\title{
Perfil pragmático de crianças surdas em diferentes fases lingüísticas
}

\section{Deaf children's pragmatic profile in different linguistic phases}

\author{
Ida Lichtig ${ }^{1}$, Maria Inês Vieira Couto ${ }^{2}$, Vanessa Nogueira Leme ${ }^{3}$
}

\begin{abstract}
RESUMO
Objetivo: O objetivo deste estudo foi verificar a interferência da fase lingüística no perfil comunicativo de crianças surdas. Métodos: Trinta crianças surdas participaram deste estudo, sendo 15 crianças em fase pré-lingüística e 15 em fase lingüística (independente da modalidade comunicativa - língua oral ou língua de sinais), na faixa etária de 41 a 59 meses. Foram analisadas 30 gravações das habilidades comunicativas da criança, em situação de interação com a examinadora. Foi utilizado o Protocolo do Perfil Pragmático das Habilidades Iniciais da Comunicação para identificar quais as habilidades comunicativas ocorridas e qual a sua frequiência de ocorrência. Resultados: Os dois grupos apresentaram basicamente as mesmas habilidades comunicativas; entretanto, o grupo Lingüístico apresentou maior freqüência de ocorrência, principalmente nas habilidades relativas a intenções comunicativas e respostas para comunicação. Conclusões: Há relação direta entre a complexidade lingüística (independente da modalidade) e o perfil comunicativo da criança surda, ou seja, a criança em fase lingüística apresenta maior freqüência de ocorrência no uso das habilidades comunicativas mais complexas e elaboradas.
\end{abstract}

Descritores: Audição; Surdez; Linguagem; Comunicação; Desenvolvimento da linguagem

\section{INTRODUÇÃO}

A linguagem pode ser definida como um sistema dinâmico e complexo de símbolos convencionais, socialmente compartilhados e organizados pela inter-relação de seus componentes funcionais: forma (fonologia, morfologia e sintaxe), conteúdo (semântica) e uso intencional (pragmática) ${ }^{(1)}$. Ela tem influência direta no desenvolvimento dos processos cognitivo, psicológico e escolar, assim como nas relações sociais do ser humano.

Pesquisas na área da surdez mostram que o desenvolvimento da forma e conteúdo da língua oral nas crianças surdas é mais lento do que em seus pares ouvintes. Em média, cinco meses do desenvolvimento da oralidade em uma criança ouvinte equivalem a um ano do desenvolvimento da criança $\operatorname{surda}^{(2-3)}$, crianças estas consideradas como bem sucedidas em programas de intervenção fonoaudiológica.

Trabalho realizado no Departamento de Fisioterapia, Fonoaudiologia e Terapia Ocupacional da Faculdade de Medicina da Universidade de São Paulo - USP - São Paulo (SP), Brasil, com financiamento concedido pela Fundação de Amparo à Pesquisa do Estado de São Paulo (FAPESP).

(1) Professora Associada do Curso de Fonoaudiologia do Departamento de Fisioterapia, Fonoaudiologia e Terapia Ocupacional da Faculdade de Medicina da Universidade de São Paulo - USP - São Paulo (SP), Brasil.

(2) Doutora, Técnica Especializada do Departamento de Fisioterapia, Fonoaudiologia e Terapia Ocupacional da Faculdade de Medicina da Universidade de São Paulo - USP - São Paulo (SP), Brasil.

(3) Pós-graduanda em Ciências da Reabilitação pela Faculdade de Medicina da Universidade de São Paulo - USP - São Paulo (SP), Brasil.

Endereço para correspondência: Ida Lichtig. R. Alberto Faria, 102, Pinheiros, São Paulo - SP, CEP 05459-000. E-mail: idalichtig@yahoo.com.br Recebido em: 28/11/2007; Aceito em: 4/6/2008
Lingüistas $^{(4-5)}$ esclarecem que existe uma analogia no processo de desenvolvimento da língua de sinais e da língua oral, pois ambas incluem a fase pré-linguística (balbucio e gestos) e a fase lingüística (os demais estágios do desenvolvimento dos componentes forma e conteúdo da linguagem).

A aquisição da linguagem pressupõe a competência lingüística de estruturas abstratas e o uso apropriado das mesmas, que é denominada pragmática ${ }^{(6)}$. O objeto de estudo da pragmática é o discurso conversacional e a maneira como os participantes, em uma situação de comunicação, processam informação verbal e não-verbal com objetivo de atingirem a comunicação efetiva $^{(7)}$.

A avaliação do perfil pragmático, por meio da observação das habilidades comunicativas, tem se mostrado como um importante meio de avaliar a competência comunicativa de crianças com atraso ou alteração no desenvolvimento da língua oral devido à perda auditiva, pois na avaliação da linguagem, baseada nas perspectivas pragmáticas, o comportamento comunicativo é categorizado independentemente se é expresso na forma verbal ou não verbal ${ }^{(8)}$. Portanto, a observação da presença das habilidades comunicativas também contribuirá para que o fonoaudiólogo tenha uma perspectiva abrangente e objetiva a respeito dos elementos que estarão em jogo no processo terapêutico ${ }^{(9)}$.

Algumas pesquisas examinaram as habilidades comunicativas de crianças com perda auditiva profunda em processo de interação ${ }^{(10-12)}$ e concluíram que, nos estágios pré-lingüístico e lingüístico inicial, independente da modalidade comunicativa, essas crianças parecem usar as mesmas habilidades comunicativas que crianças ouvintes. 
A literatura demonstra que há associação entre o atraso no desenvolvimento da linguagem e o número total de atos comunicativos das crianças surdas: as que interagem mais apresentam menos atraso no desenvolvimento da linguagem, independente da modalidade de comunicação utilizada ${ }^{(13)}$.

Em um estudo sobre o perfil geral das funções de linguagem $^{(14)}, 12$ crianças surdas de três a sete anos de idade foram distribuídas em dois grupos, de acordo com a fase lingüística: um grupo pré-linguístico e outro lingüístico com crianças usuárias da Língua Brasileira de Sinais (Libras). Para a investigação das funções de linguagem (ou habilidades comunicativas), foi adotada a classificação de Wells ${ }^{(15)}$, denominada como funções controladora, expressiva, representativa, tutelar, planejadora e social. Verificaram que as crianças, ainda que em diferentes fases de desenvolvimento, apresentavam todas as funções da linguagem, porém com diferentes perfis. As crianças em fase lingüística apresentaram como principal função da linguagem a representação, enquanto que as crianças em fase pré-linguística utilizaram a linguagem preferencialmente como função expressiva. As crianças surdas em fase pré-linguística atribuem à linguagem a função expressiva, enquanto que as crianças surdas em fase linguística, usuárias de Língua de Sinais, atribuem à linguagem a função representativa.

Em outro um estudo com crianças surdas na faixa etária entre sete e 11 anos de idade, foi verificado que elas usavam todas as funções de linguagem, sendo que, a complexidade aumentava com o aumento da faixa etária, independente da modalidade comunicativa. As crianças surdas com idade média de 11 anos e nove meses apresentaram predominantemente subfunções mais complexas, quando comparadas às crianças surdas com idade média de sete anos e seis meses ${ }^{(16)}$.

O Programa de Intervenção Fonoaudiológica em Famílias de Crianças Surdas (Piffics) ${ }^{(17)}$ apresenta o protocolo para avaliação de linguagem, no qual consta, em uma de suas partes, o perfil pragmático da comunicação, baseado no The pragmatic profile of early communications skills ${ }^{(18)}$, a fim de obter informações sobre as habilidades comunicativas de crianças surdas até sete anos de idade na interação com outros, seja no ambiente clínico ou por meio do relato dos pais.

A partir do pressuposto que crianças surdas desenvolvem as mesmas habilidades comunicativas que seus pares ouvintes, independente da modalidade utilizada na interação, com perfis diferentes de acordo com a faixa etária e a fase lingüística em que se encontram; há escassez de informações sobre as habilidades comunicativas na transição do período pré-linguístico para o lingüístico inicial, são necessários novos estudos que auxiliem o fonoaudiólogo na realização de um procedimento de avaliação mais fácil e rápido, bem como na compreensão do desenvolvimento de linguagem em crianças surdas, que estão na transição da fase pré-linguística para a lingüística inicial.

Este estudo tem como objetivo verificar a interferência da fase lingüística (independente da modalidade - língua oral ou língua de sinais) no perfil comunicativo de crianças surdas.

\section{MÉTODOS}

Este estudo é parte da pesquisa analisada e aprovada pela Comissão de Pesquisa do Departamento de Fisioterapia, Fono- audiologia e Terapia Ocupacional da Faculdade de Medicina da Universidade de São Paulo (protocolo 192/02). Todos os responsáveis pelas crianças surdas assinaram o Termo de Consentimento Livre e Esclarecido.

\section{Casuística}

Participaram deste estudo 30 crianças surdas, que foram alocadas em dois grupos, sendo um Grupo Pré-Linguístico (GPL) e um Grupo Lingüístico (GL), após terem sido submetidas à avaliação de linguagem. Para tanto, utilizou-se o protocolo do Piffics ${ }^{(17)}$, no qual foram avaliados os aspectos morfológicos e sintáticos da produção das crianças, independente da modalidade comunicativa (língua de sinais ou língua oral). Utilizou-se o cálculo do Mean Length of Longest Utterances (MLU-L - Extensão Média das Emissões Mais Longas), que é uma versão simplificada do MLU ${ }^{(19)}$. Para utilizar este procedimento, foi adaptada a esses critérios estabelecidos a seleção das três emissões lingüísticas mais longas produzidas por cada criança (tanto em língua oral como em língua de sinais) ${ }^{(20)}$. Sabe-se que, nos primeiros anos de vida, as crianças surdas filhas de pais ouvintes geralmente encontram-se na fase prélingüística ou raramente produzem estruturas com uma palavra ou sinal (MLU-L variando entre 0 e 1,0$)^{(17)}$.

Foram selecionadas crianças de acordo com os seguintes critérios: ser portadora de perda auditiva neurossensorial de grau severo ou profundo, na melhor orelha e ser filha de pais ouvintes. Foram excluídas as crianças com problemas neurológicos, com distúrbios de comportamento e com má formação crânio-facial.

O GPL era composto por 15 crianças com idade entre 42 e 59 meses de idade (média de 49,7 meses e desvio padrão de $5,6) ; 53,3 \%$ do sexo masculino e $46,7 \%$ do feminino; $66,7 \%$ com grau de perda auditiva neurossensorial profunda e $33,3 \%$ com grau severo na melhor orelha; 53,3\% estavam adaptadas às próteses auditivas e $80 \%$ não frequientavam terapia fonoaudiológica. As crianças do GPL apresentaram MLU-L variando entre 0 e 1,0 .

O GL era composto por 15 crianças com idade entre 41 e 58 meses de idade (média de 51,2 meses e desvio padrão de 4,9); $60 \%$ eram do sexo masculino e $40 \%$ do sexo feminino; $73,3 \%$ com grau de perda auditiva neurossensorial profunda e $26,7 \%$ de grau severo na melhor orelha; $66,7 \%$ estavam adaptadas às próteses auditivas e 13,3\% freqüentavam a terapia fonoaudiológica. As crianças do GL apresentaram MLU-L variando entre 1,0 e 4,6 (média de 1,9 e desvio padrão de 0,9 ), sendo que três eram usuárias da língua oral e as demais da língua de sinais.

\section{Material e equipamento}

Foram utilizados os seguintes materiais e equipamentos: Protocolo de Avaliação da Comunicação da Criança Surda Primeira Parte: Perfil Pragmático da Criança Surda ${ }^{(17)}$, filmadora Panasonic (modelo NV-VJ66PN) com tripé, videocassete Panasonic (modelo NVDH665BR) e folha de registro para cada criança para computar a frequiência de ocorrência e a modalidade das habilidades comunicativas. 
O protocolo "Perfil pragmático da criança surda" (17) é uma adaptação do "Perfil das habilidades iniciais da comunicação"(18). Trata-se de uma lista de habilidades comunicativas compreendidas em quatro secções, no qual deve ser registrada a ocorrência de cada habilidade. Por ser um material do tipo checklist para avaliar as habilidades pragmáticas, ou seja, assinalar a ocorrência ou não de uma habilidade, não há necessidade de dados normativos.

O protocolo é composto por 33 habilidades comunicativas, mas para o presente estudo, foram utilizadas 25 delas, ou seja, as três primeiras secções:

A) Intenções comunicativas: fornece informações sobre variedades e formas de intenções comunicativas expressas pela criança: 1) a criança ganha a atenção do interlocutor?, 2) a criança solicita algo?, 3) a criança rejeita?, 4) a criança cumprimenta?, 5) a criança demonstra emoção?, 6) a criança reconhece algo e nomeia?, 7) a criança comenta sobre objeto ou ação?, 8) a criança informa sobre algo que aconteceu?

B) Respostas para a comunicação: fornece informações sobre as respostas da criança diante do interlocutor: 9) o interlocutor obtém a atenção da criança?, 10) a criança responde ao interlocutor?, 11) a criança compreende gestos?, 12) a criança compreende o que o interlocutor está falando?, 13) a criança compreende a intenção do falante?, 14) a criança antecipa algum fato?, 15) a criança responde com diversão?, 16) a criança tenta negociar?

C) Interação e conversação: fornece informações sobre as interações ocorridas entre a criança e o interlocutor: 17) a criança inicia interação?, 18) há manutenção da interação ou conversação?, 19) a criança é compreendida - emite com inteligibilidade?, 20) a criança compartilha conhecimento?, 21) a criança faz reparos na conversação?, 22) a criança faz pedidos de clareza?, 23) a criança termina uma interação?, 24) a criança reage quando está observando casualmente uma conversa? e 25) a criança participa de uma conversação que outras pessoas estão tendo?

\section{Procedimento}

Por tratar-se de um estudo retrospectivo e descritivo, as 30 fitas de vídeo contendo as gravações das avaliações de linguagem foram transcritas e analisadas. Cada gravação continha o registro da atividade de interação entre a dupla, ou seja, uma criança surda e uma adulta (surda fluente em língua de sinais ou ouvinte fluente em língua oral - conforme a modalidade de comunicação preferencial da criança) não familiar a essa. Nesta atividade, foram utilizados brinquedos em miniatura em atividade semi-estruturada, nos dois grupos de crianças.

Foi realizada a análise da gravação de 15 minutos iniciais de interação lúdica entre cada criança surda e a interlocutora adulta, para verificar a frequiência de ocorrência das habilidades comunicativas referidas no protocolo. Durante a transcrição da fita houve a colaboração de uma intérprete de Libras.

As freqüências de ocorrências de cada habilidade comunicativa foram tabuladas e submetidas à análise estatística, utilizando-se o teste qui-quadrado para verificar as diferenças na distribuição das freqüências de ocorrências das 23 habilidades comunicativas nos dois grupos (GPL e GL). Foi considerado significativo o valor de $\mathrm{p}<0,05$.

Para a análise das outras duas habilidades comunicativas, Interesse em interações (10) e Mantendo uma interação ou conversação (18), o teste qui-quadrado não pode ser aplicado devido à dificuldade para quantificar a ocorrência destas habilidades, pois estão inseridas em outras habilidades. Nestes casos, verificou-se a presença, total ou parcial, ou ausência destas habilidades para cada criança, sendo que estas ocorrências foram classificadas como: freqüentemente, às vezes ou nunca ocorreu. Posteriormente, foi realizada a análise descritiva destes dados.

\section{RESULTADOS}

Foram analisadas 3844 ocorrências das habilidades comunicativas do GL e 2471 ocorrências do GPL. Observou-se que os dois grupos apresentaram perfis de comportamento comunicativo semelhantes: maior número de ocorrência de habilidades referentes a "Respostas para comunicação", seguido de "Intenções comunicativas" e "Interação e conversação". O GL apresentou maior número de ocorrência das habilidades comunicativas na secção "Interação e Conversação" e menor ocorrência na secção "Respostas para comunicação". A Figura 1 apresenta a distribuição das ocorrências das habilidades comunicativas dos dois grupos em cada secção do protocolo.

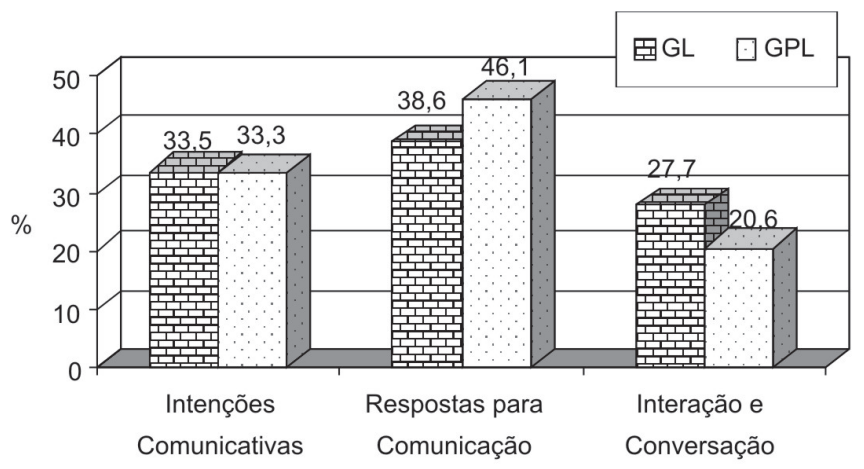

Habilidades Comunicativas

Legenda: $\mathrm{GL}$ = grupo linguístico; GPL = grupo pré-linguístico

Figura 1. Distribuição das ocorrências das habilidades comunicativas do GL e do GPL

Para descrever e especificar o perfil pragmático dos dois grupos nas três secções, foi verificada a distribuição das frequiências de ocorrência das habilidades comunicativas para cada grupo (Tabelas 1, 2 e 3).

A ocorrência das habilidades da secção "Intenções comunicativas" mais frequientes $(1,2,5$ e 7$)$ foram as mesmas para os dois grupos; porém, a baixa incidência das habilidades 4 (a criança cumprimenta?) e 8 (a criança informa sobre algo que aconteceu?) impossibilitou o estudo estatístico das mesmas. Houve predomínio estatisticamente significante $(\mathrm{p}<0,0001)$ na frequiência de ocorrência das habilidades de direcionamento da atenção (1), nomeação (6) e comentário (7) no GL em relação ao GPL; e maior frequiência de ocorrência das habilidades autoexpressão e auto afirmação (5) e rejeitando (3) no GPL em relação ao GL. Não houve diferença significante da habilidade 
Tabela 1. Distribuição da freqüência de ocorrências das habilidades comunicativas que compõem a secção "Intenções comunicativas" pelos dois grupos

\begin{tabular}{|c|c|c|c|c|c|c|}
\hline \multirow[t]{2}{*}{ Habilidades } & \multicolumn{2}{|c|}{$\mathrm{GL}$} & \multicolumn{2}{|c|}{ GPL } & \multicolumn{2}{|c|}{ Total } \\
\hline & $\mathrm{N}$ & $\%$ & $\mathrm{~N}$ & $\%$ & $\mathrm{~N}$ & $\%$ \\
\hline 1-Direcionamento de atenção & 522 & 40,3 & 273 & 33,2 & 795 & 37,6 \\
\hline 2-Solicitação & 116 & 9,0 & 91 & 11,1 & 207 & 9,8 \\
\hline 3-Rejeitando & 6 & 0,5 & 22 & 2,7 & 28 & 1,3 \\
\hline 4-Cumprimentos & 3 & 0,2 & 2 & 0,2 & 5 & 0,2 \\
\hline 5-Auto-expressão e auto-afirmação & 174 & 13,4 & 241 & 29,3 & 415 & 19,6 \\
\hline 6-Nomeação & 91 & 7,0 & 24 & 2,9 & 115 & 5,4 \\
\hline 7-Comentário & 380 & 29,3 & 169 & 20,6 & 549 & 25,9 \\
\hline 8-Dando informação & 3 & 0,2 & 0 & 0,0 & 3 & 0,1 \\
\hline Total & 1295 & 100,0 & 822 & 100,0 & 2117 & 100,0 \\
\hline
\end{tabular}

Legenda: $\mathrm{GL}$ = grupo linguístico; $\mathrm{GPL}$ = grupo pré-linguístico. Teste de Qui-quadrado: $\mathrm{p}<0,0001$ *

Tabela 2. Distribuição da freqüência de ocorrências das habilidades comunicativas que compõe "Respostas para comunicação" pelos dois grupos

\begin{tabular}{|c|c|c|c|c|c|c|}
\hline \multirow[t]{2}{*}{ Habilidades } & \multicolumn{2}{|c|}{ GL } & \multicolumn{2}{|c|}{ GPL } & \multicolumn{2}{|c|}{ Total } \\
\hline & $\mathrm{N}$ & $\%$ & $\mathrm{~N}$ & $\%$ & $\mathrm{~N}$ & $\%$ \\
\hline 1-Obtendo atenção da criança & 163 & 11,0 & 143 & 12,5 & 306 & 11,7 \\
\hline 2- Compreensão de gestos & 219 & 14,7 & 218 & 19,1 & 437 & 16,6 \\
\hline 3-Reconhecimento da emissão anterior & 642 & 43,2 & 409 & 35,8 & 1051 & 40,0 \\
\hline 4-Compreensão e intenção do falante & 303 & 20,4 & 175 & 15,3 & 478 & 18,2 \\
\hline 5-Atencipação & 0 & 0,0 & 0 & 0,0 & 0 & 0,0 \\
\hline 6-Respondendo com divertimento & 125 & 8,4 & 163 & 14,3 & 288 & 11,0 \\
\hline 7-Respostas para "não" e negação & 33 & 2,2 & 33 & 2,9 & 66 & 2,5 \\
\hline Total & 1485 & 100,0 & 1141 & 100,0 & 2626 & 100,0 \\
\hline
\end{tabular}

Legenda: $\mathrm{GL}$ = grupo linguístico; GPL = grupo pré-linguístico. Teste de Qui-quadrado: $p<0,0001$ *

2 (a criança solicita algo?) entre os grupos.

As habilidades mais frequentes da secção "Respostas para a comunicação" $(9,11,12,13$ e 15) foram as mesmas para os dois grupos; contudo, a habilidade 14 (a criança antecipa algum fato?) apresentou baixa incidência e, por este motivo, não foi tratada estatisticamente. Houve o predomínio estatisticamente significante $(\mathrm{p}<0,0001)$ da freqüência de ocor- rência das habilidades reconhecimento da emissão anterior (12) e compreensão da intenção do falante (13) no GL; e da habilidade respondendo com divertimento (15) no GPL. Não houve predomínio das habilidades 9 (o interlocutor obtém a atenção da criança?), 11 (a criança compreende gestos?) e 16 (a criança tenta negociar?) entre os grupos.

As habilidades comunicativas da secção "Interação e

Tabela 3. Distribuição da freqüência de ocorrências das habilidades comunicativas que compõe "Interação e conversação" pelos dois grupos

\begin{tabular}{|c|c|c|c|c|c|c|}
\hline \multirow[t]{2}{*}{ Habilidades } & \multicolumn{2}{|c|}{ Lingüístico } & \multicolumn{2}{|c|}{ Pré Lingüístico } & \multicolumn{2}{|c|}{ Total } \\
\hline & $\mathrm{N}$ & $\%$ & $\mathrm{~N}$ & $\%$ & $\mathrm{~N}$ & $\%$ \\
\hline 1-Iniciando interação & 481 & 45,2 & 238 & 46,9 & 719 & 45,7 \\
\hline 2-Inteligibilidade & 570 & 53,6 & 262 & 51,6 & 832 & 52,9 \\
\hline 3-Pressuposições e conhecimentos compartilhados & 0 & 0,0 & 1 & 0,2 & 1 & 0,1 \\
\hline 4-Reparos conversacionais & 3 & 0,3 & 2 & 0,4 & 5 & 0,3 \\
\hline 5-Pedido por clareza & 10 & 0,9 & 5 & 1,0 & 15 & 1,0 \\
\hline 6-Terminando uma interação & 0 & 0,0 & 0 & 0,0 & 0 & 0,0 \\
\hline 7-Ouvindo casualmente uma conversação & 0 & 0,0 & 0 & 0,0 & 0 & 0,0 \\
\hline 8-Participando de uma conversação & 0 & 0,0 & 0 & 0,0 & 0 & 0,0 \\
\hline Total & 1064 & 100,0 & 508 & 100,0 & 1572 & 100,0 \\
\hline
\end{tabular}

Legenda: GL = grupo linguístico; GPL = grupo pré-linguístico. Teste de Qui-quadrado: p=0,7920 
Tabela 4. Distribuição do percentual das habilidades 10 e 18 nos dois grupos de crianças surdas

\begin{tabular}{lcccccc}
\hline Habilidades & \multicolumn{2}{c}{ Freqüentemente } & \multicolumn{2}{c}{ Às vezes } & \multicolumn{2}{c}{ Nunca } \\
\cline { 2 - 7 } & GPL & GL & GPL & GL & GPL & GL \\
\hline 1- Interesse em interações & $80 \%$ & $93,4 \%$ & $20 \%$ & $6,6 \%$ & $0 \%$ & $0 \%$ \\
2- Mantendo uma interação ou conversação & $73,3 \%$ & $66,7 \%$ & $26,7 \%$ & $33,3 \%$ & $0 \%$ & $0 \%$ \\
\hline
\end{tabular}

Legenda: $\mathrm{GL}$ = grupo linguístico; $\mathrm{GPL}$ = grupo pré-linguístico

conversação", ilustradas na Tabela 3, mais freqüentes foram as mesmas $(17,19$ e 22) e sem predomínio ( $\mathrm{p}=0,7920)$ da freqüência de ocorrência entre os dois grupos. Ressalta-se que, as habilidades 20, 21, 23, 24 e 25 foram pouco incidentes e consequentemente não foram submetidas à análise estatística.

As habilidades comunicativas: interesse em interações (10) e mantendo uma interação ou conversação (18) foram analisadas qualitativamente como: freqüente, às vezes ou nunca, conforme ilustra a Tabela 4. Verificou-se nos dois grupos a freqüente manifestação das duas habilidades.

\section{DISCUSSÃO}

Com o objetivo de verificar a interferência da fase lingüística (independente da modalidade - língua oral ou língua de sinais) no perfil comunicativo de crianças surdas, foram avaliadas 30 crianças, na faixa etária de 41 a 59 meses.

Durante os 15 minutos iniciais de interação livre entre as duplas, obteve-se um total de amostra de habilidades comunicativas suficiente para análise estatística da maioria das habilidades, conforme ilustra a Figura 1. O GL e o GPL apresentaram desempenho semelhante nas três secções do protocolo das habilidades comunicativas utilizadas neste estudo. A frequiência de ocorrência das habilidades da secção "Respostas para comunicação" foi maior que a da secção "Intenções comunicativas" que, por sua vez, foi maior que as da "Interação e conversação".

A habilidade comunicativa pressuposições e conhecimentos compartilhados (20) manteve apenas uma ocorrência no GPL e terminando uma interação (23) não ocorreu. Isto sugere que a interlocutora não criou oportunidades para que tais funções comunicativas pudessem ocorrer. De acordo com a literatura ${ }^{(17,21)}$, a situação clínica não permite coletar todos os aspectos delineados no perfil pragmático num espaço de tempo restrito e informações complementares devem ser obtidas com os pais ou responsáveis pela criança, por meio de questionários. Ressalta-se que a interlocutora adulta utilizou estilo comunicativo do tipo não diretivo e não controlador, na interação com as crianças surdas. Este estilo propiciou o aparecimento da maioria das habilidades comunicativas expressas pelas crianças surdas. Estes fatos evidenciam a importância do treino do fonoaudiólogo na condução da avaliação das habilidades pragmáticas.

As habilidades comunicativas ouvindo casualmente uma conversação (24) e participando de uma conversação (25) não ocorreram, pois a interação foi apenas entre a interlocutora adulta e a criança, não sendo possível provocar situações de conversação com outras pessoas. Sugere-se que, nas próximas pesquisas, nas quais houver apenas dois interlocutores, essas habilidades não sejam avaliadas.
Verificou-se que em 13 habilidades comunicativas não houve diferença estatisticamente significante entre os dois grupos de crianças, sugerindo que não existe relação direta entre a fase lingüística e a ocorrência destas habilidades. São elas: 2) a criança solicita algo?, 4) a criança cumprimenta?, 8) a criança informa sobre algo que aconteceu?, 9) o interlocutor obtém a atenção da criança?, 10) a criança responde ao interlocutor?, 11) a criança compreende gestos?, 14) a criança antecipa algum fato?, 16) a criança tenta negociar?, 17) a criança inicia interação?, 18) há manutenção da interação ou conversação?, 19) a criança é compreendida - inteligibilidade?, 21) a criança faz reparos na conversação? e 22) a criança faz pedidos de clareza?

Os dados do presente estudo corroboram com os de estudos anteriores ${ }^{(14,22)}$ que afirmam que as crianças surdas, sem outros comprometimentos associados à surdez e independente da modalidade comunicativa preferencial que utilizam, podem apresentar todas as funções comunicativas; porém, os níveis de complexidade podem ser diferentes. A criança surda apresenta competência comunicativa. Contudo, a limitação da quantidade e da qualidade da comunicação dos dois grupos de crianças deste estudo provavelmente está relacionada ao lento progresso no desenvolvimento das habilidades lingüísticas, seja na modalidade auditivo-oral ou viso-espacial ${ }^{(23)}$.

Nas Tabelas 1, 2 e 3, verificou-se que o GPL apresentou um número significativamente superior de algumas funções comunicativas quando comparadas ao GL. São elas: 3) a criança rejeita?, 5) a criança demonstra emoção? e 15) a criança responde com divertimento?. Tais resultados sugerem que há relação direta entre a fase pré-linguística (independente da modalidade) e a criança surda utilizar mais as funções comunicativas que expressem emoções.

Segundo a literatura ${ }^{(24)}$, a ausência de uma língua interfere negativamente na forma de manifestação das habilidades comunicativas. As crianças surdas em fase pré-linguística comunicam-se preferencialmente por meio da função expressiva, que manifesta sentimentos e atitudes, em detrimento da função representativa, que discute postulados e eventos e obtém informações, gerando uma redução qualitativa nas interações com seus interlocutores ${ }^{(1)}$.

A literatura relata que as diferenças na qualidade e quantidade de interações comunicativas vivenciadas pela criança, podem interferir na velocidade de seu desenvolvimento e domínio lingüísticos ${ }^{(15)}$. Por sua vez, estas interações permitem um processo constante de troca de informações e estimulam a comunicação e o desenvolvimento intelectual da criança, conduzindo-a no processo de desenvolvimento da linguagem $^{(25)}$.

Nas Tabelas 1, 2 e 3, verificou-se que o GL apresentou um número significativamente superior de algumas funções 
comunicativas quando comparadas ao GPL. Foram as seguintes funções: 1) a criança ganha a atenção do interlocutor?, 6) a criança reconhece algo e nomeia?, 7) a criança comenta sobre objeto ou ação?, 12) a criança compreende o que o interlocutor está falando?, 13) a criança compreende a intenção do falante? Tais resultados sugerem que há relação direta entre a complexidade lingüística (independente da modalidade) e a criança surda utilizar mais as habilidades comunicativas que expressem uma participação mais efetiva na conversação. Tais habilidades são mais complexas, elaboradas e dependem do uso de uma língua (oral ou de sinais). Vale ressaltar a importância do direcionar a atenção e ganhar a atenção do interlocutor para se comunicar e de reconhecer a emissão e compreender a intenção do falante, as quais possibilitam a compreensão e a manutenção da comunicação.

Os achados deste estudo trazem implicações importantes para os programas de intervenção fonoaudiológica com a criança surda. Uma delas é a valorização das habilidades comunicativas não lingüísticas das crianças surdas: a partir da conscientização do profissional e dos pais sobre as possibilidades comunicativas da criança, o fonoaudiólogo poderá focar apropriadamente a oportunidade de a criança utilizar aquelas habilidades comunicativas de forma lingüística mais complexa. Outra implicação seria a análise das habilidades comunicativas de crianças surdas com outros comprometimentos associados: a freqüência da ocorrência das habilidades comunicativas indicará a existência de atraso ou desvio no desenvolvimento de linguagem. E, por fim, a contribuição para análise da evolução lingüística no processo terapêutico fonoaudiológico no contexto comunicativo.

\section{CONCLUSÕES}

Com o presente estudo pôde-se concluir que:

- As crianças surdas, em distintas fases lingüísticas (prélinguística e lingüística) e em situação de interação semi-estruturada apresentam a maioria das habilidades comunicativas.

- As crianças em fase lingüística apresentam maior freqüência de uso das habilidades comunicativas, evidenciando a importância da língua (oral ou de sinais) para garantir uma comunicação efetiva.

- Ambos os grupos, Lingüístico e Pré-Linguístico, apresentam maior freqüência de ocorrência de habilidades referentes a Respostas para Comunicação, seguido de Intenções Comunicativas e Interação e Conversação.

- As habilidades comunicativas predominantes no Grupo Lingüístico são mais complexas e elaboradas do que as habilidades predominantes no grupo Pré-Linguístico.

\begin{abstract}
Purpose: The aim of this study was to ascertain and to assess the interference of deaf children's linguistic level in their communicative abilities. Methods: Thirty deaf children participated in this study, 15 in the pre-linguistic phase and 15 in the linguistic phase, independent of the communicative modality used - either oral or sign language. Their age ranged from 41 to 59 months. Thirty videotape recordings of the deaf children's communicative abilities assessment during their interaction with the examiner were analyzed. The Communicative Abilities Pragmatic Profile was used in order to identify the communicative abilities and their frequency of occurrence. Results: Both groups basically presented the same communicative abilities; however, the linguistic group presented higher frequency of occurrence, especially in the abilities regarding communicative intentions and responses for communication. Conclusions: There is a direct relationship between linguistic complexity (independent of any kind of modality) and the deaf child's communicative profile, that is, the linguistic level child presents higher frequency of occurrence of more complex and elaborated communicative abilities.
\end{abstract}

Keywords: Hearing; Deafness; Language; Communication; Language development

\title{
REFERÊNCIAS
}

1. Owens RE. Language development: an introduction. Boston: Allyn and Bacon; 1996.

2. Miyamoto RT, Svirsky MA, Robbins AM. Enhancement of expressive language in prelingually deaf children with cochlear implants. Acta Otolaryngol. 1997;117(2):154-7.

3. Robbins AM, Svirsky MA, Kirk KI. Children with implants can speak, but can they communicate? Otolaryngol Head Neck Surg. 1997;117(3 Pt 1):155-60.

4. Quadros RM, Karnopp LB. Língua de sinais brasileira: estudos lingüísticos. Porto Alegre: Artes Médicas; c2004.

5. Woll B. Development of signed and spoken language. In: Gregory S, Knight P, McCracken W, Powers S, Watson L. editors. Issues in deaf education. London: David Fulton Publishers; 1998. p.58-68.
6. Dore J. A pragmatic description of early language development. J Psycholinguist Res. 1974;3(4):343-50.

7. Befi-Lopes DM, Cattoni DM, Almeida RC. Avaliação de aspectos da pragmática em crianças com alteração no desenvolvimento da linguagem. Pro-Fono. 2000;12(2):39-47.

8. Nicholas JG, Geers AE. Communication of oral deaf and normally hearing children at 36 months of age. J Speech Lang Hear Res. 1997;40(6):1314-27.

9. Fernandes FDM. Autismo infantil. In: Fernandes FDM, Pastorello LM, Scheuer CI. Fonoaudiologia em distúrbios psiquiátricos da infância. São Paulo: Lovise; 1996. p.17-29.

10. Curtiss S, Prutting CA, Lowell EL. Pragmatic and semantic development in young children with impaired hearing. J Speech Hear Res. 
1979;22(3):534-52.

11. Litchig I, Couto MIV, Gomes MSR, Mecca FFN, Akiyama R, Woll B. O perfil pragmático das habilidades de comunicação de crianças ouvintes e portadoras de deficiência auditiva. In: IV Congresso Internacional de Fonoaudiologia/ III Encontro Ibero-Americano de Fonoaudiologia; 1999 Out 14-16; São Paulo. Anais. São Paulo: SBFa; 1999. p.136.

12. Couto MIV, Lichtig I. Efeitos da amplificação sonora sobre as modalidades comunicativas utilizadas pelos pais. Pro-Fono. 2007;19(1):75-86.

13. Janjua F, Woll B, Kyle J. Effects of parental style of interaction on language development in very young severe and profound deaf children. Int J Pediatr Otorhinolaryngol. 2002;64(3):193-205.

14. Mecca FFDN, Cárnio MS, Lichtig I. Perfil das funções de linguagem em crianças surdas na idade de três a sete anos. Pro-Fono. 2002;14(3):44958 .

15. Wells G. Language development in the pre-school years. Cambridge: Cambridge University Press; 1985.

16. Mecca FFDN. Investigação das funções da linguagem, da teoria da mente, do vocabulário e do desempenho escolar de alunos surdos [tese]. São Paulo: Faculdade de Educação da Universidade de São Paulo; 2005.

17. Lichtig I, Couto MIV, Mecca FFDN. Avaliando as habilidades comunicativas e lingüísticas de crianças surdas. In: Lichtig I, organizador. Programa de intervenção fonoaudiológica em famílias de crianças surdas (PIFFICS). Barueri: Pró-Fono; 2004. p.39-80.
18. Dewart H, Summers S. The pragmatic profile of early communications skills. Windsor: NFER- Nelson; 1994.

19. Brown RA. First language: the early stages. Massachusetts: Harvard University Press; 1973.

20. Woll B, Wirz S, Lichtig I. Programa de atendimento a pais de crianças portadoras de deficiência auditiva com e sem linguagem oral. Technical project report submitted and funded by FAPESP, CNPq and British Council. 1996.

21. Katayama ET, Lichtig I, Couto MIV. Comportamento comunicativo da criança surda: observação no ambiente clínico e familiar. In: $21^{\circ}$. Encontro Internacional de Audiologia; 2006 Abr 20-23; Bauru. Anais [CD Rom]. Bauru; 2006.

22. Lichtig I, Couto MIV, Souza LP, Ideriha PN. Estratégias de comunicação utilizadas pelas mães para transmitir tarefas para crianças ouvintes e surdas por suas mães. Rev Soc Bras Fonoaudiol. 2001;6(1):39-45.

23. Lederberg AR, Everhart VS. Conversations between deaf children and their hearing mothers: pragmatic and dialogic characteristics. J Deaf Stud Deaf Educ. 2000;5(4):303-22.

24. Cárnio MS, Gomes MSR, Mecca FFDN, Couto MIV, Lichtig I, Carvalho RMM. Disponibilidade para atividades de leitura e escrita das crianças surdas e ouvintes de três a cinco anos de idade. Pro-Fono. 2000;12(2):67-72

25. Vygotsky LS. Pensamento e linguagem. Trad. de Jéferson Luiz de Camargo. São Paulo: Martins Fontes; 1987. 